\title{
Présentation de \\ la série thématique « Trafic intracellulaire $\gg$
}

> Le transport intracellulaire est une fonction essentielle de la cellule eucaryote et l'étude de ses mécanismes est aujourd'hui l'un des domaines les plus actifs de la recherche en biologie cellulaire. C'est donc un sujet qui justifiait largement qu'une série thématique de mises au point y soit consacrée dans médecine/sciences.

Des avancées considérables ont été réalisées ces dernières années dans la description à l'échelle moléculaire des principales voies de transport, dans l'étude des mécanismes de tri et d'adressage des protéines et des lipides, et dans la compréhension des processus complexes et étroitement contrôlés qui permettent à la cellule de maintenir à tout moment l'intégrité fonctionnelle de ses compartiments, de s'adapter à un nouvel environnement ou de répondre à des stimulus variés.

Un grand nombre de gènes, maintenant identifiés, sont directement impliqués dans le transport intracellulaire. Comme on pouvait l'escompter, des mutations de ces gènes sont retrouvées dans un nombre croissant de maladies. On peut citer comme exemples de pathologies associées à des défauts du transport intracellulaire la mucoviscidose, de nombreuses maladies liées au fonctionnement anormal des lysosomes et des peroxysomes, et certains retards mentaux liés au chromosome $X$. Un exemple particulièrement remarquable est celui du syndrome de Griscelli, une maladie autosomique récessive touchant les enfants et se caractérisant par une immunodéficience associée à un albinisme partiel des cheveux et de la peau. Une série de travaux récents, réalisés en particulier par l'équipe de Geneviève de Saint-Basile et Alain Fisher de l'Hôpital Necker (Paris, France)

$[1,2]$, a montré que les bases moléculaires du

1. Pastural $\varepsilon$, Barrat FJ, Dufourcq-Lagelouse R, et al. Griscelli disease maps to chromocome $15 q$ and is associated with mutations in the myosin-Va gene. Nat Genet $1997 ; 16: 289-92$.

2. Ménasché $G$, Pastural $\varepsilon$, Feldmann J, et al. Mutations in RAB27A cause Griscelli syndrome associated with haemophagocytic syndrome. Nat Genet $2000 ; 25: 173-6$.

3. Goud B. Le transport vésiculaire des cellules eucaryotes est contrôlé par les GTP-ases. Med Sci $1992 ; 8$ : 326-33.

4. Goud B. How Rab proteins link motors to membranes. Nat Cell Biol $2002 ; 4$ : ع77-8. syndrome de Griscelli sont des mutations affectant soit le fonctionnement d'une petite protéine $G$ (appartenant à une grande famille de protéines régulatrices du transport intracellulaire, les protéines Rab) [3], soit le fonctionnement d'un moteur moléculaire de la famille des myosines et permettant le mouvement des mélanosomes le long du cytosquelette d'actine. La caractérisation des mutations associées à ces différentes maladies a bien évidemment stimulé la recherche fondamentale et, réciproquement, les progrès de celle-ci ont été essentiels à la compréhension de ces maladies comme, par exemple, la mise en évidence que les petites protéines $\mathrm{G}$ de la famille Rab interagissent directement ou indirectement avec les moteurs moléculaires [4].
Cnrs UMR 144, Institut Curie, 26, rue d'Ulm, 75248 Paris Cedex 05, France.

Bruno.Goud@curie.fr

Une série d'articles décrivant l'état de nos connaissances sur les principaux aspects du transport intracellulaire débute dans ce numéro. Le texte de Andrée Tixier-Vidal (« Les compartiments membranaires de la cellule eucaryote $»)$ situe dans une perspective historique les données de base indispensables à la compréhension des recherches actuelles sur les mécanismes moléculaires du transport intracellulaire, celui de Bruno Antonny (« Contrôle de l'assemblage des manteaux protéiques COP par les petites protéines $G$ Arf et Sar ») explique comment les manteaux protéiques nécessaires à la formation des vésicules de transport s'associent aux membranes des compartiments cellulaires. Dans les prochains numéros, le texte de Thierry Galli et al. sera consacré aux mécanismes de la fusion membranaire, un processus complexe et finement contrôlé qui permet aux vésicules de transport de « reconnaître » spécifiquement leurs membranes cibles et de fusionner avec elles pendant les processus de transport ; celui de Nicolas Morel et Jean-Marc Philippe (« La libération des neuromédiateurs : le double-jeu de la VATPase ») présentera le cas particulier de la fusion des vésicules synaptiques avec la membrane présynaptique dans les neurones. Les numéros suivants présenteront les données récentes sur les voies d'entrée dans les cellules des macromolécules du milieu extracellulaire, voies qui se révèlent plus complexes que prévu (Alexandre Benmerah et Christophe Lamaze : « Endocytose : chaque voie compte ») et sur le rôle des lipides dans le transport, domaine de recherche particulièrement actif et en plein essor (Anne Schmidt : « Rôle de la modification des lipides dans la biogenèse des vésicules et la fission »). Enfin, cette série thématique sera clôturée par la mise au point de Serge Benichou et Alexandre Benmerah sur les mécanismes d'entrée des virus, en particulier le VIH et le virus de l'Herpès (« Virus et voie d'endocytose »), et par un texte de synthèse et de prospective dans lequel seront présentés les enjeux majeurs de la recherche dans le domaine du transport intracellulaire dans les prochaines années.

Lorsque ce « feuilleton » aura atteint son terme, les lecteurs de $\mathrm{m} / \mathrm{s}$ auront à leur disposition, nous l'espérons, un dossier actualisé et didactique sur ce domaine exemplaire à l'interface de la biologie fondamentale et de la pathologie. < 